\title{
Guperar HIV broadly neutralizing antibody targets
}

\author{
Constantinos Kurt Wibmer ${ }^{\mathrm{a}, \mathrm{b}}$, Penny L. Moore ${ }^{\mathrm{a}, \mathrm{b}, \mathrm{c}}$, and Lynn Morris ${ }^{\mathrm{a}, \mathrm{b}, \mathrm{c}}$
}

\begin{abstract}
Purpose of review
To provide an update on neutralizing antibody targets in the context of the recent HIV-1 envelope trimer structure, describe new antibody isolation technologies, and discuss the implications of these data for HIV-1 prevention and therapy.
\end{abstract}

\section{Recent findings}

Recent advances in B-cell technologies have dramatically expanded the number of antibodies isolated from HIV-infected donors with broadly neutralizing plasma activity. These, together with the first high-resolution crystal and cryo-electron microscopy (cryo-EM) structures of a cleaved, prefusion HIV-1 trimer, have defined new regions susceptible to neutralization. This year, three epitopes in the gp 120-gp4 1 interface were structurally characterized, highlighting the importance of prefusion gp41 as a target. Similar to many other broadly neutralizing antibody epitopes, these new antibodies define a target that is also highly glycan dependent. Collectively, the epitopes for broadly neutralizing antibodies now reveal a continuum of vulnerability spanning the length of the HIV-1 envelope trimer.

\section{Summary \\ Progress in the last year has provided support for the use of rationally stabilized whole HIV-1 trimers as immunogens for eliciting antibodies to multiple epitopes. Furthermore, the increasing number of broad and potent antibodies with the potential for synergistic/complementary combinations opens up new avenues for preventing and treating HIV-1 infection.}

\section{Keywords}

glycans, gp 120-gp41 interface, HIV-1 envelope, neutralizing antibody epitopes

\section{INTRODUCTION}

The HIV-1 envelope (Env) glycoprotein spike mediates viral entry, and is the sole target of neutralizing antibodies. The entry-mediating form exists as a trimer composed of three host receptor binding gp120 molecules, noncovalently linked to three gp41 transmembrane fusion proteins. gp120 is heavily glycosylated and shielded by the hypervariable regions (loops V1-V5, the $\alpha 2$ helix, and $\beta 14$ sheet), whereas gp41 is more conserved, less solvent exposed, and less glycosylated. As a result of host immune pressures Env is the most diverse of all HIV proteins with up to $30 \%$ variation between different genetic subtypes. Amino acid substitution, insertions/deletions, and glycan shifting occur predominantly in the variable regions that are most easily accessed by neutralizing antibodies. The dominant neutralizing antibody response is therefore strain specific, however over the course of HIV-1 infection most individuals develop antibodies with some level of cross-reactivity [1]. Those with the greatest breadth have been the source of new broadly neutralizing antibodies (bNAbs) [2]. Characterizing the epitopes of these bNAbs has led to high resolution structures of the HIV-1 Env trimer [3,4,5"'], allowing us to more clearly define sites of vulnerability that might be exploited for HIV-1 vaccine design and antibody mediated therapy.

\section{TECHNOLOGIES FOR THE ISOLATION OF NEW BROADLY NEUTRALIZING ANTIBODIES}

The first bNAbs to HIV-1 (b12, 2G12, 2F5, and 4E10) were isolated by phage display or B-cell immortalization, selected for binding to Env peptides or

\footnotetext{
${ }^{a}$ Centre for HIV and STIs, National Institute for Communicable Diseases (NICD), National Health Laboratory Service (NHLS), ${ }^{b}$ Faculty of Health Sciences, University of the Witwatersrand, Johannesburg and ${ }^{\mathrm{C} C e n t r e}$ for the AIDS Programme of Research in South Africa (CAPRISA), University of KwaZulu-Natal, Durban, South Africa

Correspondence to Prof. Lynn Morris, Centre for HIV and STIs, National Institute for Communicable Diseases, National Health Laboratory Service (NHLS), Private Bag X4, Sandringham 2131, Johannesburg, South Africa. Tel: +27 11386 6332; fax: +27 11386 6456; e-mail: lynnm@ nicd.ac.za
}

Curr Opin HIV AIDS 2015, 10:135-143

DOI: $10.1097 / \mathrm{COH} .0000000000000153$ 


\section{KEY POINTS}

- Technological advances have significantly improved our ability to map plasma bNAb specificities and isolate relevant $\mathrm{mAbs}$.

- The gp 120-gp41 interface is revealed as a new bNAb target.

- At least five broadly defined bNAb targets exist that form a continuum of vulnerability spanning the length of the HIV-1 Env trimer.

- New mAb combinations show unprecedented levels of cross-clade coverage and potency providing opportunities for preventive and therapeutic applications of bNAbs.

- Structure and immunogenicity studies of bNAb epitopes on the HIV-1 Env trimer may foster the design of better vaccines able to elicit bNAbs.

monomeric proteins, and generally limited in breadth and/or potency. The ability to culture memory B cells, together with high-throughput neutralization assays that allowed for direct functional screening, led to the isolation of several new antibodies targeting novel quaternary structure specific epitopes, as well as more potent antibodies to previously identified sites $\left[6-8,9^{\prime \prime}, 10^{-"}\right]$. New bNAbs to previously known targets (but possessing greater breadth and potency) have also been identified using structure-guided methods to design sorting antigens for labelling B cells by flow cytometry $[11,12]$. Unlike B-cell culture this technique does not rely on potent neutralization to identify bNAbs, but it is limited by the specific mode of recognition. More recently, quaternary structure specific bNAbs have been isolated using native, cleaved, prefusion trimers as sorting antigens, which appear to preferentially bind neutralizing antibodies [13"-].

The successful isolation of bNAbs has been aided by first mapping the neutralization specificities in donor plasma, to tailor an appropriate selection technique [14,15]. In addition bioinformatics approaches have been used to predict specificities and design targeted approaches for the isolation of bNAbs [16-18]. Once a B-cell lineage has been identified the use of next-generation sequencing to mine the repertoire allows for literally hundreds of related variants to be identified [19-22]. A major obstacle of next-generation sequencing however is the inability to identify naturally occurring heavychain and light-chain antibody pairs. This was overcome when Georgiou et al. devised a method of pairing heavy-chain and light-chain PCR products prior to sequencing [23]. Information on the targets for bNAbs, as well as neutralization, sequence, and structural data on the monoclonal antibodies (mAbs) that have been isolated is being extensively catalogued into two new publically available databases: CATNAP on the LANL website (http:// www.hiv.lanl.gov/components/sequence/HIV/neu tralization/main.comp) and bNAber [24], providing useful resources for the field.

\section{BROADLY NEUTRALIZING ANTIBODY TARGETS}

The isolation of exceptionally broad and potent bNAbs has enabled the identification of five roughly defined targets on the HIV-1 Env, such as the V2 site, the N332 supersite, the CD4 binding site (CD4bs), the gp120-gp41 interface, and the membrane proximal external region (MPER). Identifying multiple bNAbs with similar epitopes has pinpointed minimal sites of vulnerability, whose recognition confers the greatest neutralization breadth. However as discussed below, new bNAbs with novel epitopes have revised our understanding of how these distinct sites partially merge in the context of the trimer.

\section{THE V2 SITE}

The V2 site at the trimer apex is formed from the converging, sequence conserved regions of the V1V2 domain and the V3 loop $[3,4,25]$. It is protected by densely packed glycans (particularly those at positions N156 and N160) and the hypervariable loops V1 and V2 [26]. Access to the underlying peptide epitope is only possible by antibodies with unusually long (between 26 and 39 amino acids), anionic heavy chain complementarity determining region loop three (CDR-H3) [7,14,26]. Anti-V2 bNAbs generally bind poorly to monomeric gp120 or scaffolded V1V2s $[7,14]$. In the case of the prototypical V2 antibody PG9, this quaternary specificity was partially explained by the fact that the antibody binds to N160 glycans from two separate protomers $[27,28]$. However, for some relatives of the CAP256-VRC26 lineage which targets a similar epitope, broad neutralization was not dependent on the N160 glycan [14]. Despite these differences, the actual peptide epitope determined by mutagenesis is minimal for both the PG9 and CAP256-VRC26 antibodies, made up of a short mostly cationic stretch of seven amino acids (position 165-171). For PG9 the underlying peptide comprises less than $25 \%$ of the epitope, with the rest of the epitope predominantly formed by the glycans at N156 and N160 $[7,14,26,29]$. The conserved nature of these glycans, and the small peptide footprint, likely contributes to the breadth of this class of antibodies. 


\section{THE N332 SUPERSITE}

The N332 supersite is composed of a number of overlapping glycan-dependent epitopes [30]. V3 epitopes lie structurally proximal to the V2 site [4], and are the most well described within the N332 supersite. Antibodies targeting V3 show a similar mechanism to V2 site recognition, in that they access a minimal eight residue peptide epitope between positions 323 and 330 via long (20-26 amino acids) CDR-H3s [31]. Two such antibodies, PGT121 and PGT128, are highly dependent on the glycans at positions N301 and N332 [6], but somatic variants of PGT121 also depend on glycans in V1 (N137) and V2 (N156) [4]. In this way PGT121-like antibodies can recognize a different side of the N156 glycan that is critical to most anti-V2 bNAbs (Fig. 1). PGT130 was isolated from the same donor as PGT128, but represents an alternate branch of the B-cell lineage that preferentially recognizes a glycan at N334 [6,32]. The N334 and N332 glycans are mutually exclusive, and thus in both donors somatic variants have evolved to recognize different immunotypes of the V3 site, indicative of the role for viral diversification in driving bNAb maturation, that ultimately accounts for total plasma neutralization breadth $[32,33]$.

bNAb PGT135 defines a second epitope in the N332 supersite that does not involve V3, but rather contacts amino acids at the base of the V4 loop [30]. This $\mathrm{mAb}$ binds predominantly to the apolar face of the N332 glycan (unlike PGT128), and recognizes additional glycans at positions N295, N386, and N392 [30]. A third epitope, defined by the bNAb 2G12 overlaps the PGT135 epitope but unusually does not involve any peptide contact (Fig. 1). Rather, $2 \mathrm{G} 12$ uses a rare variable heavy $(\mathrm{VH})$ chain domain swap to create a large paratope capable of binding to the terminal sugars of four glycans on the silent face of gp120 (N295, N332, N339, and N392) [34]. Thus, while 2 G12 does not actually penetrate the glycan shield, it does share the recognition of key glycans with PGT135. The isolation of additional bNAbs with specificities similar to PGT135 and 2G12 will help to better define the vulnerabilities within this supersite.

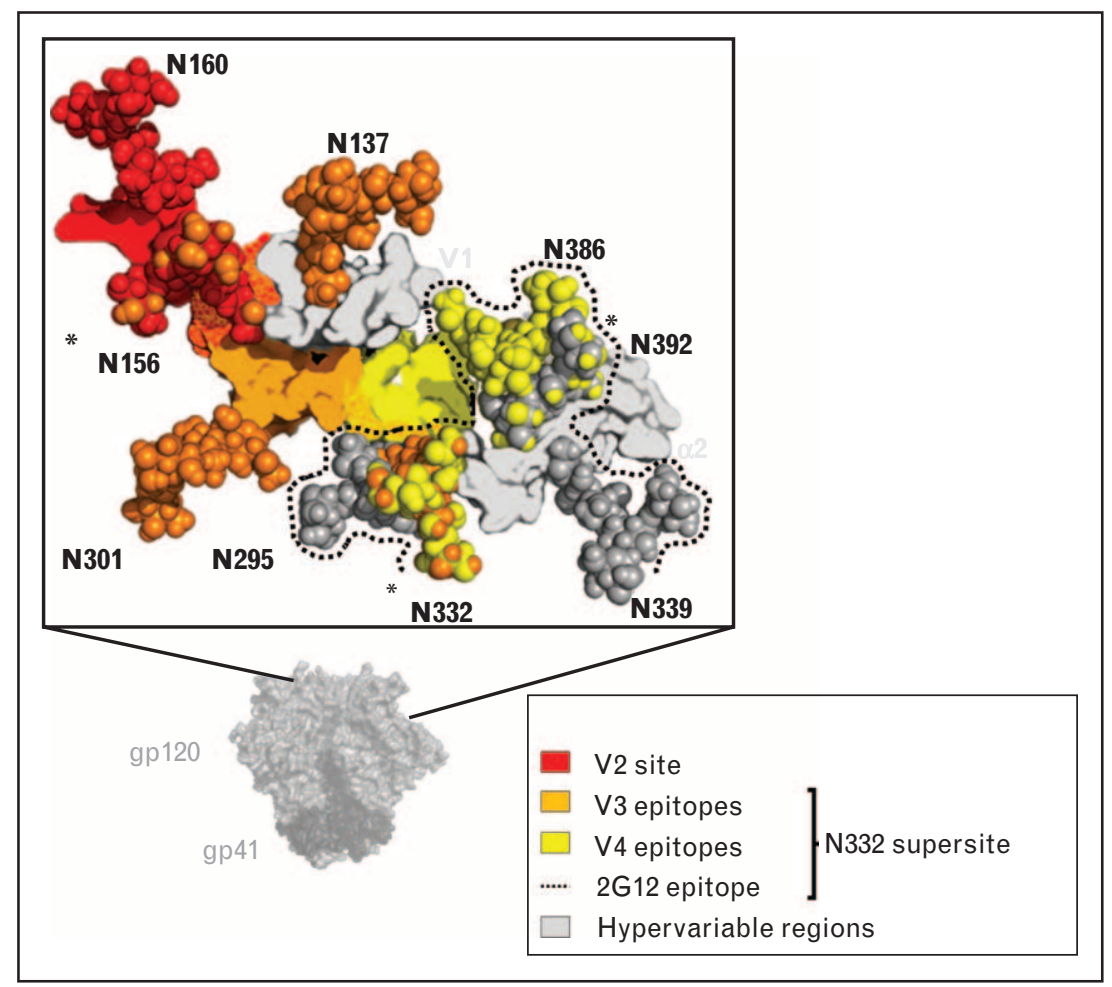

FIGURE 1. The V2 site overlaps with the N332 supersite. The HIV-1 envelope (Env) trimer is shown in light (gp120) and dark (gp41) grey surface view. An expanded graphic of the V2 and N332 sites is shown in the zoomed out box. Epitopes in V2 (red), V3 (orange), or V4 (yellow) are defined as residues within $5 \AA$ of broadly neutralizing antibodies (bNAbs) PG9, PGT122/PGT128, or PGT135, respectively. Regions of overlap between epitopes are shown with mottled colours red/orange for V2-V3, and orange/yellow for V3-V4. Key glycans are shown as spheres, labelled, and coloured according to their epitopes. Glycans predicted to be a part of the 2G12 epitope are coloured dark grey, and bordered with a dotted line. Glycans N156, N332, and N392 that are bound by bNAbs targeting different epitopes are asterisked. Proximal hypervariable regions $\mathrm{V} 1$ and $\alpha 2$ are shown in light grey. Figure based on protein databank accession code 4TVP. 


\section{THE CD4 BINDING SITE}

In contrast to bNAbs targeting V2 or N332, antibodies targeting the CD4bs generally make minimal glycan contacts. Only one mode of recognition at the CD4bs has been extensively described, that of VH1-2 or VH1-46 derived bNAbs, typified by VRC01 and 8ANC131. The germline precursors of these bNAbs have specific genetic signatures that mimic CD4 binding, such as R71 in the heavy chain that, like R59 of CD4, interacts with D368 in the highly conserved CD4 binding loop of gp120 [12,35]. VH12 bNAbs also have unusually short CDR-L3 loops to avoid clashes with the glycan at position N276 in the D loop of gp120 [36-38]. Conversely the bNAb HJ16 derived from the VH3-30 gene does not interact with D368, and is entirely dependent on the glycan at position N276 for neutralization, defining a second subsite within the CD4bs $[39,40]$. This glycan is sometimes bound by VRC01-like antibodies, but is not critical to their neutralization [41]. HJ16 and other non-VH1-2/1-46 derived CD4bs bNAbs have long CDR-H3 loops important for their binding, but are still limited in their angle of approach to gp120 by extensive glycosylation and the quaternary nature of the trimer $[15,42]$. Cryoelectron microscopy (cryo-EM) structures have shown that some CD4bs bNAbs such as PGV04 may actually interact with the glycans that closely border the CD4bs (N276, N363, and N386) as well as the N301 glycan from the V3 loop of an adjacent protomer [3]. These data also suggest contact between the positively charged amino acids in V3, and an anionic insertion in the PGV04 heavy-chain framework region 3 (FWR3). The contribution of these additional contacts to neutralization is unclear considering many CD4bs bNAbs induce a conformation of gp120 that would rearrange the V1V2 and V3 loops. Nonetheless the complexity of the CD4bs has previously been underestimated.

\section{THE gp120-gp41 INTERFACE}

The N276 glycan described above is also the target for bNAb 8ANC195 (Fig. 2), recently shown to bind an epitope in the gp120-gp41 interface [43"']. This $\mathrm{mAb}$ is dependent on glycans at positions N234 and N276, and uses a four amino acid insertion to thrust the Fab heavy-chain FWR3 between these two

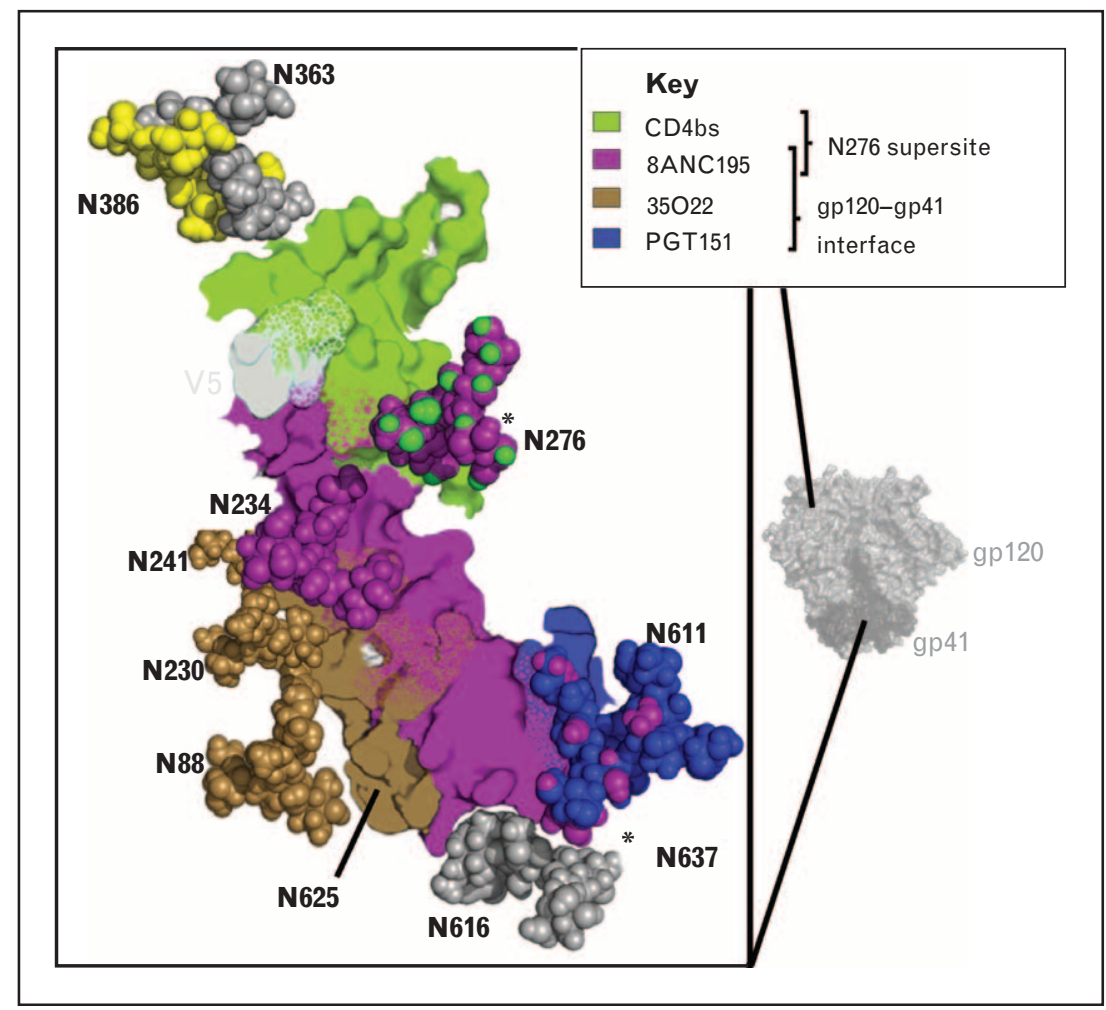

FIGURE 2. The CD4bs adjoins the gp 120-gp41 interface. The HIV-1 envelope trimer is shown in light (gp 120) and dark (gp41) grey surface view. An expanded graphic of the CD4bs and gp120-gp41 interface sites are shown in the zoomed out box. Epitopes for VRCO1 (green), 8ANC195 (purple), 35022 (brown), or PGT151 (blue) are defined as residues within $5 \AA$ of each respective broadly neutralizing antibody (bNAb). Key glycans are shown as spheres, labelled, and coloured according to the epitopes they comprise. Regions that make up more than one epitope are shown with mottled colours, and glycans that are bound by bNAbs targeting different epitopes are asterisked. The hypervariable region V 5 is shown in light grey. 
glycans, contacting R456 at the distal most tip [43"']. The 8ANC195 CDR-H3 is 22 amino acids long, but is not used to penetrate the glycan shield. Rather the CDR-H3 folds back towards the light chain forming part of a wider paratope that makes significant contact with the 7-stranded $\beta$-sandwich (the gp41 interacting region) of gp120 [43"-]. The affinity for gp120 is sufficient that 8ANC195 was isolated using a stabilized gp120 core, but docking of the Fab-gp120 complex into the prefusion trimer revealed potential interactions between the 8ANC195 light chain and gp41 near the N637 glycan. Deep sequencing in the 8ANC195 donor showed that the stabilized core specifically selected for a single branch of the bNAb lineage. More genetically diverse antibodies, created by pairing related heavy-chain and light-chain variants, exhibited increased potencies that were attributed almost entirely to light chain-gp41 interactions [43"']. Thus, 8ANC195 was the first of several recently identified bNAbs targeting epitopes in the gp120gp41 interface $\left[9^{-"}, 10^{-"}, 43^{-"}, 44\right]$
Two new antibodies published in 2014, PGT151 and $35 \mathrm{O} 22$, were also shown to target the gp120-

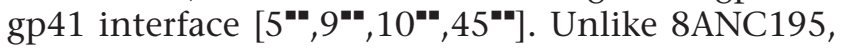
these bNAbs do not bind monomeric gp120. bNAb $35 \mathrm{O} 22$ (isolated from the same donor as MPER bNAb 10E8) binds immediately adjacent to 8ANC195 near N234 (Fig. 2), close enough to contact the sevenstranded $\beta$-sandwich via an eight amino acid FWR3 insertion [5"']. This bNAb epitope is remarkably low on the trimer, seemingly incompatible with the presence of the viral membrane, and it is speculated that certain rearrangements induced by CD4 are required for its binding [9"']. Neutralization by $35 \mathrm{O} 22$ was incompatible with glycosylation of the N625 sequon, but still critically dependent on the N625 residue [5"']. bNAb PGT151 binds between the 8ANC195 and 35O22 epitopes, over a cavity between gp160 protomers [45"']. Unlike 35O22, it has a specific requirement for cleaved trimers, and a maximum observed stoichiometry of two antigenbinding fragments (Fabs) per trimer, suggesting an

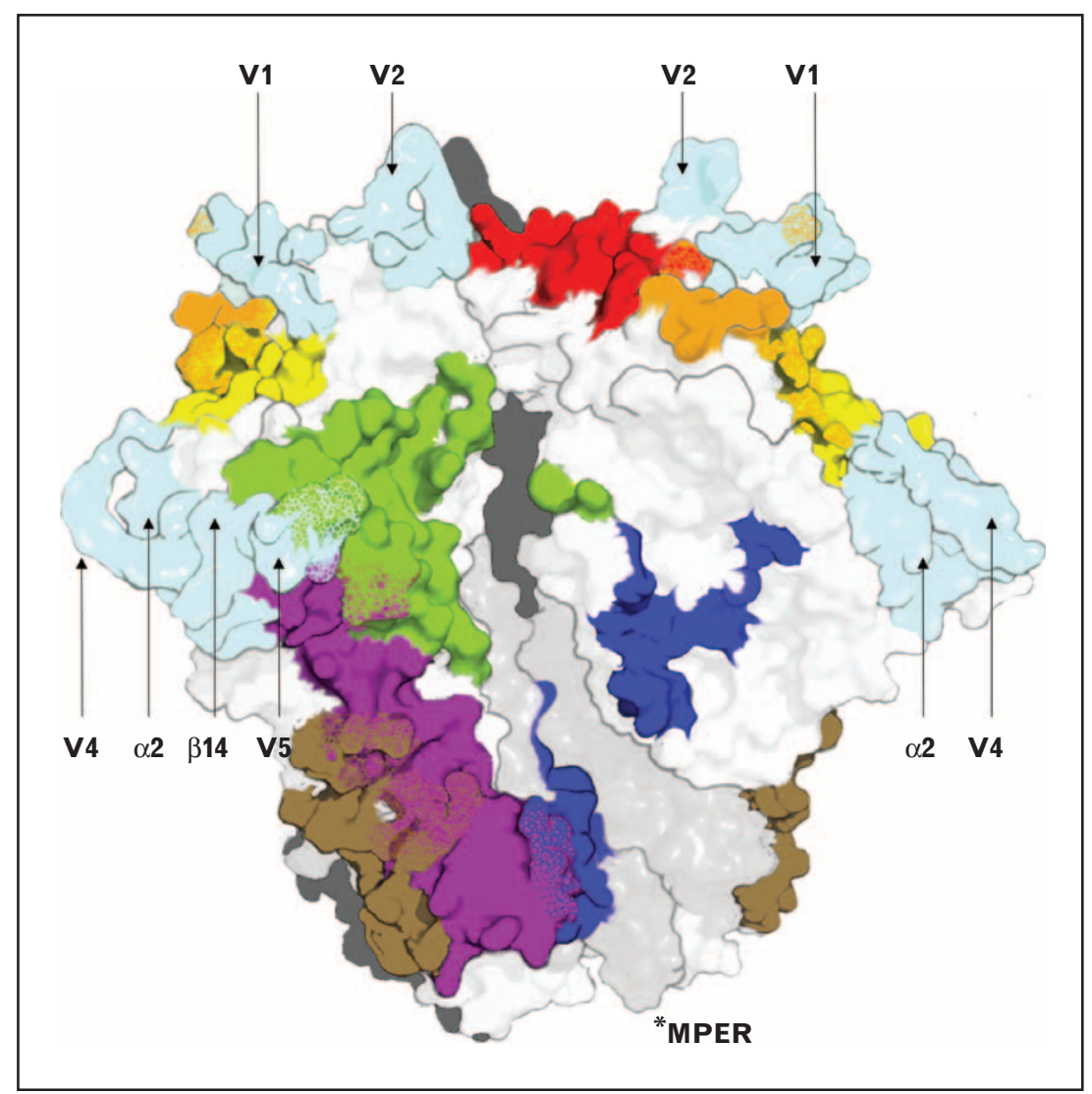

FIGURE 3. A continuum of broadly neutralizing antibody (bNAb) targets. A graphic of the HIV-1 envelope (Env) trimer is shown and surface coloured for broadly defined epitopes in V2 (red), V3 (orange), V4 (yellow), the CD4bs (green), and the gp 120-gp41 interface (brown, purple, and blue for 8ANC195, 35022, and PGT151, respectively). Overlap between these epitopes through recognition of common glycans or amino acids is indicated with mottling of the relevant colours. The highly accessible positions of hypervariable regions $\mathrm{V} 1, \mathrm{~V} 2, \alpha 2, \mathrm{~V} 4, \beta 14$, and $\mathrm{V} 5$ (coloured in cyan) are indicated with arrows. The first residue of the MPER (truncated in the HIV-1 trimer structure 4TVP) is indicated with an asterisk. 
allosteric alteration of the third binding site after the first two Fabs have bound [45"']. This binding mechanism also allows PGT151 to stabilize native, cleaved Env trimers from cell membranes. Both $35 \mathrm{O} 22$ and PGT151 have highly glycan-dependent epitopes, with $35 \mathrm{O} 22$ neutralization dependent on the glycans at N88, N230, and N241 in gp120, and neutralization of PGT151 requiring gp41 glycans N611 and N637 $\left[9^{-"^{\prime}}, 10^{\mathbf{*}}\right]$. Based on proximity, PGT151 was also predicted to interact with glycans in gp120 (N262, N276, N230, N234, N241, and N448) though these interactions are not critical for neutralization [45"']. This glycan dependence results in varied neutralization plateaus for both bNAbs. For 35O22, neutralization plateaus were improved (higher levels of maximum inhibition indicating enhanced potency) when pseudoviruses were grown in kifunensine, indicating a role for high mannose glycans in its epitope [9"']. In contrast, PGT151 could not neutralize kifunensine or 293S-grown pseudoviruses (glycosylated only with mannose rich glycans), and bound directly to triantennary and tetraantennary complex glycans $\left[10^{-"}, 45^{-"}\right]$. As virus grown in peripheral blood mononuclear cells appears to have greater proportions of complex glycan, PGT151-like antibodies may exhibit better potencies in vivo [10"'].

\section{THE MEMBRANE PROXIMAL EXTERNAL REGION}

Epitopes in the MPER of gp41 are almost exclusively contained within a linear, $\alpha$-helical stretch of amino acids that links the transmembrane domain to the ectodomain of gp41. The MPER can be divided into an N-terminal and C-terminal helix around a kink at position 674, used to define various overlapping epitopes. bNAb 2F5 binds to the N-terminal helix, Z13e1 binds to the elbow between helices, while 4E10 and 10E8 bind to an epitope in the C-terminal helix. The hydrophobic C-terminus of the MPER is partially buried in the viral membrane and is thought to play a critical role in fusion with the host membrane. It is therefore highly conserved, and antibodies $4 \mathrm{E} 10$ and $10 \mathrm{E} 8$ are some of the broadest yet identified $[8,46]$. bNAbs targeting the MPER often interact with the viral membrane via long CDR-H3 loops, which may promote higher than normal levels of autoreactivity for these types of antibodies [47]. The precise location and conformation of the MPER in the prefusion HIV-1 trimer is not yet known, and further characterization will be necessary to determine the proximity of, and relationship between, epitopes in this site and those in the gp120-gp41 interface.

\section{A CONTINUUM OF VULNERABILITY}

The increased availability of bNAbs targeting novel sites, or variants of previously described sites, has blurred the definition of distinct bNAb epitopes. For instance CD4bs antibodies, once almost exclusively classified by their sensitivity to mutations at D368, now include a new group typified by HJ16 that are largely glycan dependent and insensitive to mutations at D368 [39,40]. Similarly V2 antibodies, previously defined as being N160 glycan dependent, now also include antibodies such as CAP256-VRC26 that target the same protein epitope but are not necessarily sensitive to glycan deletion [14]. These antibodies might be described as targeting subsites within the greater CD4bs or V2 site of vulnerability. This paradigm currently does not apply to the N332dependent antibodies PGT128, PGT135, and 2G12, whose epitopes have mostly nonoverlapping peptide components (Figs. 1 and 3). One way to characterize these larger epitope clusters has been to define glycan supersites [30]. Based on these criteria, the glycan at position N276, which is the target for CD4bs antibodies (such as HJ16) and certain gp120-gp41 interface antibodies (e.g. 8ANC195) might now also be classified as a glycan supersite (Figs. 2 and 3). Other glycans, such as those at positions N156, N386, and in the newly described gp120-gp41 epitope cluster, are bound by antibodies targeting two distinct epitopes, but only form a critical component for one (Figs. 1 and 2). These glycans do not yet meet the requirements of a supersite (though isolation of additional mAbs may change this), but they may contribute towards the affinity maturation of bNAbs at both sites. Thus, it seems that bNAb epitopes form a continuum of vulnerability that includes conserved residues stretching from the trimer apex to the MPER (Fig. 3). The glycans which protect these regions, divide up the continuum into sites of vulnerability, but also because of their conservation become targets for neutralization themselves.

\section{IMPROVED NEUTRALIZATION COVERAGE}

Vaccine-induced bNAbs ideally need to protect against infection from all viral subtypes, and therefore those that target highly conserved epitopes are likely to be most effective. First-generation bNAbs, such as b12, 2F5, and 2G12, had fairly restricted breadth, and were often more effective against subtype-matched heterologous viruses [46]. More recently isolated bNAbs show much greater breadth [2], although even among the broadest bNAbs coverage (and/or potency) varies between subtypes $\left[7,9^{-"}, 14\right]$. Subtype preference was also seen using large panels of chronic sera, which showed 
increased potency of plasmas against subtypematched viruses [48]. Notably, however, this effect was reduced in older and more diversified subtypes, suggesting that the significance of this observation for vaccine design will decrease overtime [48]. Differential coverage may occur for other reasons - bNAbs to the N332-supersite are less effective against viruses with the glycan at 334 despite some promiscuity in recognition [49], a finding that may be important as the 332 glycan is less common in subtype $C$ founder viruses [50]. However, as a single antibody lineage could evolve to recognize both N332 and N334 variants (such as in the case of PGT128 and PGT130), inclusion of multiple immunotypes into vaccine design strategies may enhance coverage.

The availability of a large number of new more potent and cross-reactive mAbs has also enhanced prospects for passive immunotherapy. Early studies demonstrated that bNAbs could protect against infection in nonhuman primates and humanized mice [51-56]. However, these first-generation bNAbs were only able to transiently reduce viral loads in infected subjects [57-59]. The latest mAbs (mostly in combinations) resulted in more durable control of viremia while the $\mathrm{mAbs}$ were present, and in some cases thereafter [60-62]. Passively infused bNAbs will probably be used in combination. Indeed, mAbs targeting four different sites in double, triple, and quadruple combinations showed 98-100\% coverage [63]. In addition to coverage, potency is likely to be important, with bNAbs engineered for greater in-vitro potency associated with protection at lower plasma levels [64]. Lack of potency may be a particular concern for bNAbs with relatively low neutralization plateaus (incomplete neutralization even at high concentrations) such as 35O22, PGT151, and many V2-dependent antibodies. Ongoing human clinical trials will allow assessment of the dose of bNAb that is required (for protection and immunotherapy) and whether systemic infusion protects mucosal portals of infection.

\section{CONCLUSION}

The last year has brought significant advances in our understanding of the HIV-1 Env structure and its neutralization targets. New isolation technologies have allowed for less biased identification of bNAbs targeting increasingly complex epitopes. These new antibodies have provided important insights, culminating in the near complete structural delineation of the HIV-1 Env spike. However, in many instances only a single $\mathrm{mAb}$ targeting a given site is available (e.g. PGT135, 2G12, 8ANC195, PGT151, and $35 \mathrm{O} 22$ ), and these often demonstrate some level of clade preference. Many more antibodies will need to be identified to properly characterize each of these new HIV-1 vulnerabilities. Additional bNAb-trimer complex structures will be necessary to define 'hotspots' or supersites for neutralization. Using the structural data from multiple bNAb-antigen complexes for a single target site will facilitate rational approaches to display specific sites of vulnerability, as is currently being done for the CD4bs and MPER epitope scaffolds. However, the extensive overlap between bNAb targets suggests that immunogen design may benefit from efforts to include whole, stabilized, cleaved HIV-1 trimers perhaps rationally designed to expose the entire continuum of bNAb vulnerabilities while minimizing antigenicity to the hypervariable structures. The number of new bNAb targets, and our understanding of their structure have provided novel opportunities for preventing and treating HIV-1.

\section{Acknowledgements}

The authors thank Jinal Bhiman and Jay Gorman for helpful discussions.

\section{Financial support and sponsorship}

The authors receive research funding from the Centre for the AIDS Programme of Research (CAPRISA), the South African Medical Research Council (MRC) through the Flagship and SHIP programs, the National Institutes of Health (NIH) through an R01 Grant (R01 AI104387-01A1) and a U01 grant (AI116086-01), the Center for HIV/AIDS Vaccine Immunology (CHA VI grant AI067854), the Center for AIDS Vaccine Discovery (CAVD) of the Bill and Melinda Gates Foundation, the South African HIV/AIDS Research and Innovation Platform of the South African Department of Science and Technology and by a HIVRAD NIH grant (AI088610). CAPRISA was supported by NIAID, NIH, US Department of Health and Human Services (Grant U19 AI51794). P.L.M. is a Wellcome Trust Intermediate Fellow in Public Health and Tropical Medicine (Grant 089933/Z/ 09/Z). C.K.W. is supported by the Poliomyelitis Research Foundation (PRF) of South Africa and was the recipient of a Fogarty AITRP Fellowship.

\section{Conflicts of interest}

There are no conflicts of interest.

\section{REFERENCES AND RECOMMENDED \\ READING}

Papers of particular interest, published within the annual period of review, have been highlighted as:

- of special interest

-1. of outstanding interest

1. Hraber $P$, Seaman MS, Bailer RT, et al. Prevalence of broadly neutralizing antibody responses during chronic HIV-1 infection. AIDS 2014; 28:163-169.

2. Klein $\mathrm{F}$, Mouquet $\mathrm{H}$, Dosenovic $\mathrm{P}$, et al. Antibodies in HIV-1 vaccine development and therapy. Science 2013; 341:1199-1204. 
3. Lyumkis $\mathrm{D}$, Julien JP, de Val N, et al. Cryo-EM structure of a fully glycosylated soluble cleaved HIV-1 envelope trimer. Science 2013; 342:1484-1490.

4. Julien JP, Cupo A, Sok D, et al. Crystal structure of a soluble cleaved HIV-1 envelope trimer. Science 2013; 342:1477-1483.

5. Pancera $M$, Zhou T, Druz A, et al. Structure and immune recognition of trimeric n. prefusion HIV-1 Env. Nature 2014; 514:455-461.

Using $35 \mathrm{O} 22$ and PGT122, the authors of this article crystallized the Env trimer in near entirety, building on work by Julien et al., 2013 [4], by resolving gp41. These data revealed the interactions that stabilize the prefusion trimer, and showed how HIV-1 Env proteins share remarkable mechanistic similarity with respiratory syncytial virus, Ebola, and influenza. The complex with 35022 revealed binding to N625 that was incompatible with a glycan covalently linked to this side chain.

6. Walker LM, Huber M, Doores KJ, et al. Broad neutralization coverage of HIV by multiple highly potent antibodies. Nature 2011; 477:466-470.

7. Walker LM, Phogat SK, Chan-Hui PY, et al. Broad and potent neutralizing antibodies from an African donor reveal a new HIV-1 vaccine target. Science 2009; 326:285-289.

8. Huang J, Ofek G, Laub L, et al. Broad and potent neutralization of HIV-1 by a gp41-specific human antibody. Nature 2012; 491:406-412.

9. Huang J, Kang BH, Pancera M, et al. Broad and potent HIV-1 neutralization by

- a human antibody that binds the gp41-gp120 interface. Nature 2014; 515:138-142.

This article detailed the third novel gp120-gp41 epitope to be described in 2014, that of 35022 . The authors did extensive mapping to show that this antibody was sensitive to mutations in potential $N$-linked glycosylation sites not associated with PGT151 or 8ANC195. The bNAb, isolated from the same donor as $10 \mathrm{E} 8$, has an unusual angle of approach that appears to clash with the viral membrane. Like PGT151, this antibody exhibited unusual neutralization plateaus that were explained by a preference for high mannose glycan.

10. Falkowska E, Le KM, Ramos A, et al. Broadly neutralizing HIV antibodies

n. define a glycan-dependent epitope on the prefusion conformation of gp41 on cleaved envelope trimers. Immunity 2014; 40:657-668.

Shortly after publication of the 8ANC195 epitope, this article detailed a second novel gp120-gp41 interface epitope targeted by the PGT151 lineage. This was the first article to confirm a role for gp41 glycans in the epitopes of bNAbs. These antibodies have unusually low neutralization plateaus that could be explained by a preference for complex glycan.

11. Wu X, Yang $Z Y$, Li $Y$, et al. Rational design of envelope identifies broadly neutralizing human monoclonal antibodies to HIV-1. Science 2010; 329:856-861.

12. Scheid JF, Mouquet $H$, Ueberheide $B$, et al. Sequence and structural convergence of broad and potent HIV antibodies that mimic CD4 binding. Science $2011 ; 333: 1633-1637$

13. Sok $D$, van Gils $M J$, Pauthner $M$, et al. Recombinant HIV envelope trimer

I. selects for quaternary-dependent antibodies targeting the trimer apex. Proc Natl Acad Sci U S A 2014; 111:17624-17629.

This article describes for the first time the use of stabilized trimers for sorting single antigen specific B cells. Using the same donor as PGT145 they isolated somatic variants some of which had greater potency and breadth. This technological advance may make trimer specific bNAbs, or those of unknown specificity, easier to isolate in the future.

14. Doria-Rose NA, Schramm CA, Gorman J, et al. Developmental pathway for potent V1V2-directed HIV-neutralizing antibodies. Nature 2014; 509: 55-62.

15. Liao HX, Lynch $R$, Zhou $T$, et al. Co-evolution of a broadly neutralizing HIV-1 antibody and founder virus. Nature 2013; 496:469-476.

16. West AP Jr, Scharf L, Horwitz J, et al. Computational analysis of anti-HIV-1 antibody neutralization panel data to identify potential functional epitope residues. Proc Natl Acad Sci U S A 2013; 110:10598-10603.

17. Georgiev IS, Doria-Rose NA, Zhou T, et al. Delineating antibody recognition in polyclonal sera from patterns of HIV-1 isolate neutralization. Science 2013; 340:751-756

18. Lacerda M, Moore PL, Ngandu NK, et al. Identification of broadly neutralizing antibody epitopes in the HIV-1 envelope glycoprotein using evolutionary models. Virol J 2013; 10:347.

19. He L, Sok D, Azadnia $P$, et al. Toward a more accurate view of human B-cell repertoire by next-generation sequencing, unbiased repertoire capture and single-molecule barcoding. Sci Rep 2014; 4:6778.

20. Xiao M, Prabakaran $P$, Chen $W$, et al. Deep sequencing and Circos analyses of antibody libraries reveal antigen-driven selection of $\mathrm{lg} \mathrm{VH}$ genes during HIV1 infection. Exp Mol Pathol 2013; 95:357-363.

21. Zhu J, Ofek G, Yang $Y$, et al. Mining the antibodyome for HIV-1-neutralizing antibodies with next-generation sequencing and phylogenetic pairing of heavy/light chains. Proc Natl Acad Sci U S A 2013; 110:6470-6475.

22. Zhu J, Wu X, Zhang $B$, et al. De novo identification of VRC01 class HIV-1neutralizing antibodies by next-generation sequencing of B-cell transcripts. Proc Natl Acad Sci U S A 2013; 110:E4088-E4097.

23. DeKosky BJ, Ippolito GC, Deschner RP, et al. High-throughput sequencing of the paired human immunoglobulin heavy and light chain repertoire. Nat Biotechnol 2013; 31:166-169.

24. Eroshkin AM, LeBlanc A, Weekes D, et al. bNAber: database of broadly neutralizing HIV antibodies. Nucleic Acids Res 2014; 42 (Database issue):D1133-D1139.
25. Bartesaghi A, Merk A, Borgnia MJ, et al. Prefusion structure of trimeric HIV-1 envelope glycoprotein determined by cryo-electron microscopy. Nat Struct Mol Biol 2013; 20:1352-1357.

26. McLellan JS, Pancera M, Carrico C, et al. Structure of HIV-1 gp120 V1/V2 domain with broadly neutralizing antibody PG9. Nature $2011 ; 480: 336-343$.

27. Julien JP, Lee JH, Cupo A, et al. Asymmetric recognition of the HIV-1 trimer by broadly neutralizing antibody PG9. Proc Natl Acad Sci U S A 2013; 110:4351-4356.

28. Loving R, Sjoberg M, Wu SR, et al. Inhibition of the HIV-1 spike by singlePG9/16-antibody binding suggests a coordinated-activation model for its three protomeric units. J Virol 2013; 87:7000-7007.

29. Doria-Rose NA, Georgiev I, O'Dell S, et al. A short segment of the HIV-1 gp1 $20 \mathrm{~V} 1 / \mathrm{V} 2$ region is a major determinant of resistance to V1/V2 neutralizing antibodies. J Virol 2012; 86:8319-8323.

30. Kong L, Lee JH, Doores KJ, et al. Supersite of immune vulnerability on the glycosylated face of HIV-1 envelope glycoprotein gp120. Nat Struct Mol Biol 2013; 20:796-803

31. Pejchal R, Doores $\mathrm{KJ}$, Walker LM, et al. A potent and broad neutralizing antibody recognizes and penetrates the HIV glycan shield. Science 2011; 334:1097-1103.

32. Doores KJ, Kong L, Krumm SA, et al. Two classes of broadly neutralizing antibodies within a single lineage directed to the high-mannose patch of HIV Envelope. J Virol 2014; 89:1105-1118.

33. Moore PL, Williamson $\mathrm{C}$, Morris L. Virological features associated with the development of broadly neutralizing antibodies to HIV-1. Trends Microbiol 2015; in press.

34. Calarese DA, Scanlan CN, Zwick MB, et al. Antibody domain exchange is an immunological solution to carbohydrate cluster recognition. Science 2003; 300:2065-2071.

35. Zhou T, Zhu J, Wu X, et al. Multidonor analysis reveals structural elements, genetic determinants, and maturation pathway for HIV-1 neutralization by VRC01-class antibodies. Immunity 2013; 39:245-258.

36. Zhou T, Xu L, Dey B, et al. Structural definition of a conserved neutralization epitope on HIV-1 gp120. Nature 2007; 445:732-737.

37. McGuire AT, Hoot S, Dreyer AM, et al. Engineering HIV envelope protein to activate germline $B$ cell receptors of broadly neutralizing anti-CD4 binding site antibodies. J Exp Med 2013; 210:655-663.

38. Jardine J, Julien JP, Menis $\mathrm{S}$, et al. Rational HIV immunogen design to target specific germline B cell receptors. Science 2013; 340:711-716.

39. Balla-Jhagjhoorsingh SS, Corti D, Heyndrickx L, et al. The N276 glycosylation site is required for HIV-1 neutralization by the CD4 binding site specific HJ16 monoclonal antibody. PLoS One 2013; 8:e68863.

40. Wibmer CK, Bhiman JN, Gray ES, et al. Viral escape from HIV-1 neutralizing antibodies drives increased plasma neutralization breadth through sequential recognition of multiple epitopes and immunotypes. PLoS Pathog 2013; 9:e1003738.

41. Diskin R, Klein F, Horwitz JA, et al. Restricting HIV-1 pathways for escape using rationally designed anti-HIV-1 antibodies. J Exp Med 2013; 210:12351249

42. Corti D, Langedijk JP, Hinz A, et al. Analysis of memory $B$ cell responses and isolation of novel monoclonal antibodies with neutralizing breadth from HIV-1infected individuals. PLoS One 2010; 5:e8805.

43. Scharf L, Scheid JF, Lee JH, et al. Antibody 8ANC195 reveals a site of broad

nulnerability on the HIV-1 envelope spike. Cell Rep 2014; 7:785-795.

This was the first article to show that bNAbs can bridge the gap between gp120 and gp41. It included the structural characterization of 8ANC195, including a crystal complex with gp120, and a cryo-EM co-localizing gp41 with the Fab light chain.

44. Wibmer CK, Sheward D, Bhiman JN, et al. Viral escape pathways from broadly neutralising antibodies targeting the HIV envelope cleavage site enhance MPER mediated neutralisation. AIDS Res Hum Retrovir 2014; 30:A20-A21.

45. Blattner $\mathrm{C}$, Lee JH, Sliepen $\mathrm{K}$, et al. Structural delineation of a quaternary,

-1 cleavage-dependent epitope at the gp41-gp120 interface on intact HIV-1 env trimers. Immunity 2014; 40:669-680.

A structural characterization of the PGT151 lineage in complex with the HIV-1 trimer by cryo-EM, co-published with Falkowska et al., 2014 [10"']. These data largely supported the gp41 mapping from the linked article, as well as implicating additional gp120 glycans in the epitope. Interestingly PGT151 bound to the envelope trimer with a maximum stoichiometry of two Fabs.

46. Binley JM, Wrin T, Korber B, et al. Comprehensive cross-clade neutralization analysis of a panel of antihuman immunodeficiency virus type 1 monoclonal antibodies. J Virol 2004; 78:13232-13252.

47. Verkoczy L, Kelsoe G, Haynes BF. HIV-1 envelope gp41 broadly neutralizing antibodies: hurdles for vaccine development. PLoS Pathog 2014; 10:e1004073.

48. Hraber $\mathrm{P}$, Korber $\mathrm{BT}$, Lapedes AS, et al. Impact of clade, geography, and age of the epidemic on HIV-1 neutralization by antibodies. J Virol 2014; $88: 12623-12643$

49. Sok D, Doores KJ, Briney B, et al. Promiscuous glycan site recognition by antibodies to the high-mannose patch of gp120 broadens neutralization of HIV. Sci Transl Med 2014; 6:236ra63.

50. Moore PL, Gray ES, Wibmer CK, et al. Evolution of an HIV glycan-dependent broadly neutralizing antibody epitope through immune escape. Nat Med 2012; 18:1688-1692. 
51. Hessell AJ, Poignard $P$, Hunter $M$, et al. Effective, low-titer antibody protection against low-dose repeated mucosal SHIV challenge in macaques. Nat Med 2009; 15:951-954.

52. Mascola JR, Stiegler G, VanCott TC, et al. Protection of macaques against vaginal transmission of a pathogenic HIV-1/SIV chimeric virus by passive infusion of neutralizing antibodies. Nat Med 2000; 6:207-210.

53. Moldt B, Rakasz EG, Schultz N, et al. Highly potent HIV-specific antibody neutralization in vitro translates into effective protection against mucosal SHIV challenge in vivo. Proc Natl Acad Sci U S A 2012; 109:18921-18925.

54. Parren PW, Marx PA, Hessell $A J$, et al. Antibody protects macaques against vaginal challenge with a pathogenic R5 simian/human immunodeficiency virus at serum levels giving complete neutralization in vitro. J Virol 2001; 75:83408347.

55. Balazs $A B$, Chen J, Hong $C M$, et al. Antibody-based protection against HIV infection by vectored immunoprophylaxis. Nature 2012; 481:81-84.

56. Balazs $A B$, Ouyang $Y$, Hong $C M$, et al. Vectored immunoprophylaxis protects humanized mice from mucosal HIV transmission. Nat Med 2014; 20:296300.

57. Poignard $P$, Sabbe R, Picchio GR, et al. Neutralizing antibodies have limited effects on the control of established HIV-1 infection in vivo. Immunity 1999; $10: 431-438$.
58. Trkola A, Kuster H, Rusert $P$, et al. Delay of HIV-1 rebound after cessation of antiretroviral therapy through passive transfer of human neutralizing antibodies. Nat Med 2005; 11:615-622.

59. Mehandru S, Vcelar B, Wrin T, et al. Adjunctive passive immunotherapy in human immunodeficiency virus type 1-infected individuals treated with antiviral therapy during acute and early infection. J Virol 2007; 81:11016-11031.

60. Shingai $M$, Nishimura $Y$, Klein $F$, et al. Antibody-mediated immunotherapy of macaques chronically infected with SHIV suppresses viraemia. Nature 2013; 503:277-280.

61. Barouch $\mathrm{DH}$, Whitney JB, Moldt $\mathrm{B}$, et al. Therapeutic efficacy of potent neutralizing HIV-1-specific monoclonal antibodies in SHIV-infected rhesus monkeys. Nature 2013; 503:224-228.

62. Klein F, Halper-Stromberg A, Horwitz JA, et al. HIV therapy by a combination of broadly neutralizing antibodies in humanized mice. Nature 2012; 492:118122.

63. Kong $\mathrm{R}$, Louder $\mathrm{MK}$, Wagh $\mathrm{K}$, et al. Improving neutralization potency and breadth by combining broadly reactive HIV-1 antibodies targeting major neutralization epitopes. J Virol 2014; 89:2659-2671.

64. Rudicell RS, Kwon YD, Ko SY, et al. Enhanced potency of a broadly neutralizing HIV-1 antibody in vitro improves protection against lentiviral infection in vivo. J Virol 2014; 88:12669-12682. 